\title{
MARCKS phosphorylation is modulated by a peptide mimetic of MARCKS effector domain leading to increased radiation sensitivity in lung cancer cell lines
}

\author{
TIMOTHY D. ROHRBACH, ROBERT B. JONES, PATRICIA H. HICKS, \\ ALICE N. WEAVER, TIFFINY S. COOPER, NICHOLAS J. EUSTACE, EDDY S. YANG, \\ JOHN S. JARBOE, JOSHUA C. ANDERSON and CHRISTOPHER D. WILLEY
}

Department of Radiation Oncology, University of Alabama at Birmingham, Birmingham, AL 35249, USA

Received April 8, 2016; Accepted September 27, 2016

DOI: $10.3892 / \mathrm{ol} .2016 .5550$

\begin{abstract}
Lung cancer is the leading cause of cancer-associated mortality in the United States. Kinase hyperactivation is a known mechanism of tumorigenesis. The phosphorylation status of the plasma membrane-associated protein myristoylated alanine rich C-kinase substrate (MARCKS) effector domain (ED) was previously established as being important in the sensitivity of lung cancer to radiation. Specifically, when MARCKS ED was in a non-phosphorylated state, lung cancer cells were more susceptible to ionizing radiation and experienced prolonged double-strand DNA breaks. Additional studies demonstrated that the phosphorylation status of MARCKS ED is important for gene expression and in vivo tumor growth. The present study used a peptide mimetic of MARCKS ED as a therapeutic intervention to modulate MARCKS phosphorylation. Culturing A549, H1792 and H1975 lung cancer cell lines with the MARCKS ED peptide led to reduced levels of phosphorylated MARCKS and phosphorylated Akt serine/threonine kinase 1. Further investigation demonstrated that the peptide therapy was able to reduce lung cancer cell proliferation and increase radiation sensitivity. In addition, the MARCKS peptide therapy was able to prolong double-strand DNA breaks following ionizing radiation exposure. The results of the present study demonstrate that a peptide mimetic of MARCKS ED is able to modulate MARCKS phosphorylation, leading to an increase in sensitivity to radiation.
\end{abstract}

Correspondence to: Dr Christopher D. Willey, Department of Radiation Oncology, University of Alabama at Birmingham, 619 19th Street, Birmingham, AL 35249, USA

E-mail: cwilley@uab.edu

Key words: lung cancer, myristoylated alanine rich C-kinase substrate, radiation sensitivity, effector domain, peptide mimetic

\section{Introduction}

Lung cancer is the leading cause of cancer-associated mortality in the United States (1). Common molecular properties of lung cancer include hyperactivation of receptor tyrosine kinases (RTKs) (2). Phosphatidylinositol 3-kinase (PI3K) is a potent downstream RTK signaling target, which is able to phosphorylate phosphatidylinositol $(4,5)$-bisphosphate (PIP2) to generate phosphatidylinositol (3-5)-trisphosphate (PIP3) (3). PIP3 recruits and activates phosphoinositide-dependent kinase 1 and Akt serine/threonine kinase 1 (Akt) leading to a pro-growth, pro-survival phenotype (4). The plasma membrane associated protein myristoylated alanine rich C-kinase substrate (MARCKS) is able to bind to PIP2, regulating the conversion of PIP2 to PIP3 and subsequently influencing Akt activation $(5,6)$.

MARCKS contains three domains: An N-terminal myristoylation domain, an $\mathrm{MH} 2$ domain, and an effector domain (ED) (7). The ED of MARCKS is comprised of a 25 amino acid sequence containing four phosphorylatable-serine residues and 13 positively-charged lysine residues, which allow MARCKS to bind to the plasma membrane via electrostatic interactions and sequester PIP2 (8-11). The phosphorylation status of MARCKS ED is essential in regulating the subcellular localization of MARCKS (12). Following MARCKS ED phosphorylation, the electrostatic interaction with the plasma membrane is lost, allowing MARCKS to migrate into the cytoplasm, and subsequently release PIP2 (13). Following dephosphorylation, MARCKS is able to reattach to the plasma membrane (12).

Previously, lentiviral manipulation of A549 lung cancer cell lines was used to overexpress a wild-type (WT) or non-phosphorylatable (NP) MARCKS protein under the regulation of a tetracycline promoter. The NP-MARCKS construct was generated by substituting the serine residues of the ED for alanine residues. Overexpression of NP-MARCKS led to increased sensitivity to radiation and prolonged double-strand DNA breaks following treatment with ionizing radiation (14). MARCKS does not possess endogenous enzymatic activity and therefore a 25 amino acid peptide mimetic of MARCKS ED was engineered in order to modulate MARCKS 
phosphorylation (14). In the present study, the ability of MARCKS targeted therapy to influence MARCKS phosphorylation levels and increase the sensitivity of lung cancer cells to radiation was investigated.

\section{Materials and methods}

Cell culture. Human lung cancer cell lines A549, H1792 and H1975 (American Type Culture Collection, Manassas, VA, USA) were cultured in filtered (22 $\mu \mathrm{m}$ vacuum filtration; cat no. 431097; Corning, Inc., Corning, MA, USA) RPMI-1640 (Thermo Fisher Scientific, Inc., Waltham, MA, USA) with $10 \%$ fetal bovine serum (Sigma-Aldrich; Merck Millipore, Darmstadt, Germany), $1 \%$ penicillin-streptomycin and $1 \%$ GlutaMAX $^{\mathrm{TM}}$ (Thermo Fisher Scientific, Inc.). All cells were maintained at $37^{\circ} \mathrm{C}$ in $5 \% \mathrm{CO}_{2}$.

MARCKS plasmid production. A549 lung cancer cell lines were engineered to overexpress MARCKS in a tetracycline-dependent manner as described previously (14). WT-MARCKS and NP-MARCKS sequences (GenScript USA, Inc., Piscataway, NJ, USA) were cloned into a pLenti6.3/TO/V5 (ViraPower HiPerform T-REx Gateway Expression System; cat no. A11141; Invitrogen; Thermo Fisher Scientific, Inc.) lentiviral plasmid as described previously (5). The NP-MARCKS was engineered by substituting the four serine residues in the ED to alanine residues. Exogenous MARCKS was distinguishable from endogenous MARCKS by the addition of a V-5 epitope tag on the C-terminus of WT- and NP-MARCKS.

Lentiviral particle production. Lentiviral particles were produced as described previously (14). A total of $4 \mu \mathrm{g}$ lentiviral packaging plasmid psPAX2 (plasmid no. 12260), lentiviral envelope plasmid PCMV-VSV-G (plasmid no. 8454; both Addgene, Cambridge, Inc., MA, USA) and lentiviral vector plasmid, were mixed with Lipofectamine ${ }^{\circledR} 2000$ (cat no. 11668) and Opti-MEM $^{\mathrm{TM}}$ media (cat no. 11058; both Invitrogen; Thermo Fisher Scientific, Inc.). The lentiviral mixture was added to the culture media of 293FT cells (Invitrogen; Thermo Fisher Scientific, Inc.) and the media was subsequently replaced the following morning. Lentiviral supernatant was collected after $48 \mathrm{~h}$ and filtered through a $0.45 \mu \mathrm{m}$ filter, aliquoted and stored at $-80^{\circ} \mathrm{C}$ until required. Lentiviral particles were quantified using QuickTiter p24 ELISA (Cell Biolabs, San Diego, CA, USA) (5).

Stable cell line selection. A549 cells were transduced with equal amounts $(\sim 30 \mathrm{ng} / \mathrm{ml})$ of p24 quantified tetracycline-repressor (Tet-R) packaged lentiviral particles along with $8 \mu \mathrm{g} / \mathrm{ml}$ polybrene (EMD Millipore, Billerica, MA, USA). A total of $500 \mu \mathrm{g} / \mathrm{ml}$ Geneticin ${ }^{\circledR}$ (cat no. G418; Thermo Fisher Scientific, Inc.) was used to select for Tet-R positive cells. Tet-R cells were subsequently transduced with equal amounts $(\sim 30 \mathrm{ng} / \mathrm{ml})$ of p24 quantified WT- and NP-MARCKS lentiviral particles. A total of $1 \mu \mathrm{g} / \mathrm{ml}$ Blastacidin (Thermo Fisher Scientific, Inc.) was used to select for WT- and NP-MARCKS-expressing cells. MARCKS expression was induced by culturing with $2 \mu \mathrm{g} / \mathrm{ml}$ of the tetracycline homologue doxycycline overnight at $37^{\circ} \mathrm{C}(14)$.

MARCKS peptide. MARCKS peptide was used as described previously (14). MARCKS-ED tyrosine aminotransferase
(TAT) peptide was engineered by conjugating the cell permeable human immunodeficiency virus (HIV) TAT peptide via cysteine bonds to the 25 amino acid ED sequence (KKKKKRFSFKKSFKLSGFSFKKNKK) (15). The control peptide sequence was designed using the ExPAsy random protein sequence generator (www.expasy.org) using the average amino acid composition computed from Swiss-Prot (www. uniprot.org) (CEIEEHAWNTVEMFSSFPGTQLYNDA) to control for peptide size. To eliminate the effect of the positive charges, lysine and arginine residues were changed to glutamates. Peptide was added to cultures $1 \mathrm{~h}$ prior to performance of in vivo assays, including immunoblotting, RNA expression analysis, cellular survival and DNA double-strand damage quantification. A dose establishment study identified $6.25 \mu \mathrm{M}$ as the lowest effective dose (tested, 1-25 $\mu \mathrm{M}$ ), which was selected for subsequent studies.

Immunoblotting. Immunoblotting was performed as previously described by Jarboe et al (5). Mammalian protein extraction reagent lysis buffer supplemented with protease (cat no. P8340; Sigma-Aldrich; Merck Millipore) and phosphatase inhibitors (cat nos., P0044 and P5726; Sigma-Aldrich; Merck Millipore) was used to lyse cells. Protein concentration was determined by using Pierce Bicinchoninic Protein Assay kit (Thermo Fisher Scientific, Inc.) and $\sim 10 \mu \mathrm{g}$ of protein were separated via electrophoresis on an $8 \%$ SDS-PAGE gel and transferred to a polyvinylidene difluoride membrane (Immobilon; Merck Millipore). Blots were blocked in $5 \%$ bovine serum albumin (BSA) (Sigma-Aldrich; Merck Millipore) at room temperature for $1 \mathrm{~h}$ and probed with the following primary antibodies: Phosphorylated-MARCKS (cat no., ab81295), MARCKS (cat no., ab52616; both Abcam, Cambridge, UK), phosphorylated-Akt (Ser473) (cat no., D9E, 4060), Phospho-Akt (Thr308) (cat no., C31E5E, 2965), Akt (cat no., C67E7, 4691; all Cell Signaling Technology. Inc., Danvers, MA, USA). Antibodies were used at a dilution of 1:1,000 with overnight $4^{\circ} \mathrm{C}$ incubation. Additionally, actin antibody (cat no. sc-1616; Santa Cruz Biotechnology, Inc., Dallas, TX, USA) was used at a concentration of 1:5,000 and incubated overnight at $4^{\circ} \mathrm{C}$. Blots were washed 3 times for 5 min in TBST. Peroxidase-conjugated Affini Pure Goat Anti-Mouse IgG (cat no., 115-035-166) and Affini-Pure Donkey Anti-Rabbit IgG (cat no. 711-035-152) secondary antibodies (Jackson ImmunoResearch Laboratories, Inc., West Grove, PA, USA) was added at a dilution of 1:5,000 in 5\% BSA-TBST, and incubated for $1 \mathrm{~h}$ at room temperature. Enhanced chemiluminescence (ECL) using Western Lighting-Plus ECL substrate (PerkinElmer, Inc., Waltham, MA, USA) was used to visualize the western blots as described previously (14).

RNA expression analysis. RNA expression analysis was performed at the UAB Nanostring Laboratory (Birmingham, AL, USA) (www.uab.edu/medicine/radonc/en/nanostring). The nCounter ${ }^{\circledR}$ GX Human Cancer Reference kit (NanoString Technologies, Inc., Seattle, WA, USA) was used to analyze RNA expression of 230 human cancer-associated genes. MARCKS expression in A549 NP-MARCKS and A549 WT-MARCKS cells was induced by overnight incubation at $37^{\circ} \mathrm{C}$ with doxycycline, and total RNA was collected the following day. A total of $100 \mathrm{ng}$ RNA was prepared in a $30 \mu \mathrm{l}$ 
reaction volume and run on the automated nCounter system. Quality control and normalization were performed on the raw data using positive and negative control spots on the included GX Human Cancer Reference Panel chip and five housekeeping reference genes $(16,17)$.

Cell viability and survival assay. Cells were plated in black 96-well plates (cat no. 3603; Costar; Thermo Fisher Scientific, Inc.) and allowed to adhere overnight. The following morning the control or ED peptides were added. A total of $1 \mathrm{~h}$ following peptide treatment, cells in the irradiation group were subjected to 0,5 or $8 \mathrm{~Gy}$ irradiation using a $320 \mathrm{kV}$ X-ray irradiator (Kimtron Inc., Woodbury, CT, USA). A total of 4 days following peptide addition or irradiation, ATP levels were measured via the ATPlite ${ }^{\mathrm{TM}}$ Luminescence Assay System (PerkinElmer, Inc., Waltham, MA, USA) using a Synergy H1 Multi-Mode Reader (BioTek Instruments, Inc., Winooski, VT, USA).

In vivo studies. The animal protocol was provided by the University of Alabama at Birmingham's Institutional Animal Care and Use Committee. Mice were housed at $\sim 24^{\circ} \mathrm{C}$ and given free access to food and water. A $12 \mathrm{~h}$ light/dark schedule was provided for the mice. A total of 1 million WT- or NP-MARCKS-expressing A549 lung cancer cells, were injected into the flanks of $\sim 7$ week old female athymic nude mice ( $\sim 17 \mathrm{~g}$ ) (Charles River Laboratories, Hartford, CT, USA). Cells were mixed in a 1:1 mixture of PBS:Matrigel ${ }^{\circledR}$ (BD Biosciences, San Jose, CA, USA) to a final volume of $50 \mu \mathrm{l}$ and loaded into a $1 \mathrm{ml}$ syringe with a $25 \mathrm{gx} 5 / 8$ " needle (Monoject $^{\mathrm{TM}}$ Tuberculin Syringe; cat no. \#8881501640; Covidien, Dublin, Ireland) (18). When tumors were palpable, mouse food was subsequently supplemented with doxycycline (cat no. TD.05125; Harlan Laboratories, Madison, WI, USA) (19). WT- and NP-MARCKS expression was induced with doxycycline-supplemented mouse food for 7 days prior to tumor volume calculation. Tumor volume was measured using Vernier calipers using the formula: (Length $\mathrm{x}$ width $\left.{ }^{2}\right) / 2(20,21)$. Values are presented as the mean fold change in tumor volume \pm standard error of the mean (SEM). Mice were euthanized with $\mathrm{CO}_{2}$ inhalation as set forth by institutional guidelines.

Double-strand DNA damage quantification. Following overnight induction of MARCKS expression, lung cancer cells were treated with the control or ED peptides $1 \mathrm{~h}$ prior to $8 \mathrm{~Gy}$ irradiation. As described previously, cells were fixed and stained with an anti-phosphorylated- $\gamma \mathrm{H} 2 \mathrm{AX}-\mathrm{S} 139$ antibody and counterstained with DAPI (14). $\gamma \mathrm{H} 2 \mathrm{AX}$ staining foci marking DNA double-strand breaks were analyzed using an EVOS FL digital inverted fluorescence microscope (Thermo Fisher Scientific, Inc.). Positive events were defined as $\geq 10$ foci per cell, and positive and negative controls were included in all experiments (14,22-24).

Statistical analysis. Statistics calculations and data graphing was performed using GraphPad Prism (GraphPad Software, Inc., La Jolla, CA, USA). Analysis of variance followed by the Bonferroni correction post hoc test was used to quantify DNA damage, and a Student's $t$-test was performed to analyze survival assays. All statistics were two-sided and $\mathrm{P}<0.05$ was considered to indicate a statistically significant difference. Values are presented as the mean \pm SEM.

\section{Results}

Phosphorylation status of MARCKS ED influences lung cancer biology. MARCKS ED is known to have multiple biological functions; however, limited information is available regarding the involvement of MARCKS in lung cancer biology (25). Reversible phosphorylation of MARCKS ED is important in regulating the function and subcellular localization of MARCKS $(10,26)$. In the present study, A549 lung cancer cells were engineered to express WT- or NP-MARCKS under the regulation of a tetracycline inducible promoter. Western blotting (Fig. 1A) confirmed that A549 cells were able to overexpress WT- and NP-MARCKS in a tetracycline/doxycycline-inducible manner. Due to the ability of MARCKS to regulate multiple signaling cascades (25), the effect of MARCKS phosphorylation on gene expression was analyzed. Using the nCounter ${ }^{\circledR}$ GX Human Cancer Reference kit, differences in gene expression between A549 cells overexpressing WT- and NP-MARCKS were investigated. A total of 19 genes exhibited a $>1.5$ fold increase in expression in the WT-MARCKS cell line compared to the NP-MARCKS cell line, including siah E3 ubiquitin protein ligase 1, androgen receptor, matrix metalloproteinase (MMP) 1, platelet derived growth factor subunit A, protein tyrosine kinase 7, notch 1, MYB proto-oncogene, transcription factor, caspase 10 , Wnt family member 10B, MMP9, retinoic acid receptor alpha, WD repeat domain 87 , v-myc avian myelocytomatosis viral oncogene neuroblastoma derived homolog, B-cell CLL/lymphoma 6 , leukemia inhibitory factor, fibroblast growth factor receptor 4, S100 calcium binding protein A4, cytochrome P450 family 1 subfamily A member 1 and Fos proto-oncogene, AP-1 transcription factor subunit (Fig. 1B). Several of these genes are associated with tumorigenesis and cancer progression (27-31). Cell viability assays were performed to investigate if the ED phosphorylation status was important in lung cancer proliferation. The ATPlite assay indicated significantly increased levels of ATP in the WT-MARCKS A549 cells compared with the NP-MARCKS cell line (Fig. 1C; P=0.002), suggesting that non-phosphorylated MARCKS impedes cell viability. In addition, in vivo mouse studies were used to assess the ability of MARCKS ED phosphorylation status to influence lung cancer growth. WT- and NP- MARCKS A549 cells were injected into the flanks of athymic nude mice. Mouse food was supplemented with doxycycline to induce the expression of WT- and NP- MARCKS (19). The change in tumor volume was compared between the WT- and NP-MARCKS A549 cells over a period of 40 days. WT-MARCKS transfected mice exhibited a greater increase in tumor volume compared with NP-MARCKS transfected mice (Fig. 1D). By modeling the data to a nonlinear regression fit for exponential growth, a significant difference between the WT- and NP-MARCKS tumors $(\mathrm{P}<0.0001)$ was observed. The results of the present study suggest that the phosphorylation status of MARCKS ED influences gene expression, viability and tumor growth.

MARCKS ED provides a potential therapeutic intervention to alter lung cancer biology. Previously, a 25-amino 
A

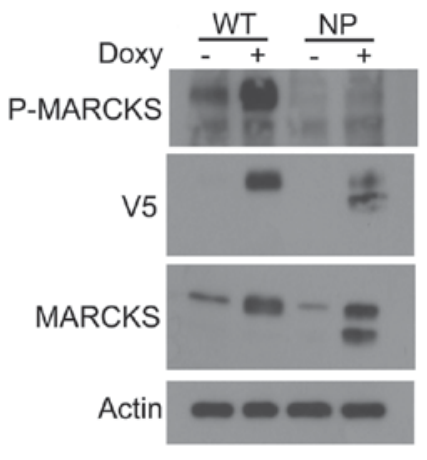

C

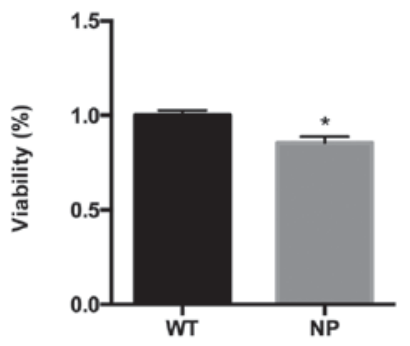

B

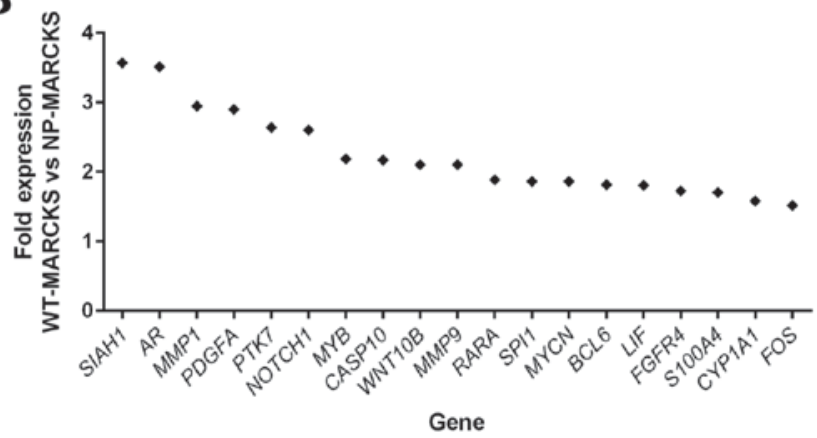

D

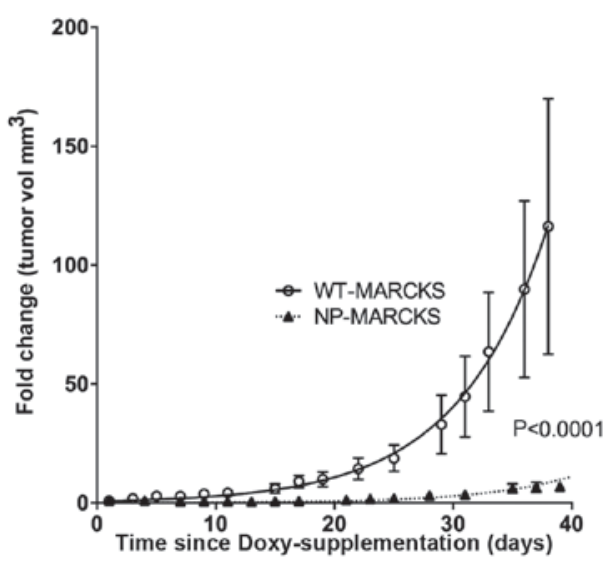

Figure 1. Phosphorylation status of MARCKS ED impacts tumorigenicity. (A) Western blot analysis of A549 lung cancer cells engineered to express WT- and NP-MARCKS under the regulation of a Doxy-inducible promoter. A total of $2 \mu \mathrm{g} / \mathrm{ml}$ of Doxy was used to induce the expression of WT- and NP-MARCKS. Antibodies were used to probe for phosphorylated MARCKS, MARCKS, V5 and broad range actin. (B) The nCounter ${ }^{\circledR}$ GX Human Cancer Reference kit identified 19 genes that had a >1.5 fold increased expression in the WT-MARCKS A549 cells compared with the NP-MARCKS A549 cells. (C) ATPlite cell viability assay measured ATP levels in WT- and NP-MARCKS expressing-A549 cells 4 days following Doxy treatment. Fluorescence levels were normalized to A549 WT-MARCKS cells ( $\mathrm{P}<0.05)$. (D) Change in tumor volume of A549 WT- and NP-MARCKS expressing tumors in athymic nude mice. WT- and NP-MARCKS expression was induced with doxycycline-supplemented mouse food for 7 days prior to tumor volume calculation. Tumor volumes were calculated over a period of 40 days. Values are presented as a linear regression of the mean \pm standard error of the mean (n=9-10; 1 mouse died unexpectedly prior to tumor formation). MARCKS ED, myristoylated alanine rich C-kinase substrate's effector domain; WT, wild-type; NP, non-phosphorylatable; Doxy, doxycycline; $\mathrm{P}$, phosphorylated.

acid peptide mimetic of MARCKS ED was used to alter lung cancer biology (14). The 25-amino acid ED peptide was conjugated to the cell permeable TAT-peptide, which allowed the ED mimic to enter the cell. Swiss-Prot software calculated a control peptide comprised of 25 random amino acids to control for peptide length. The previous investigation observed changes in cell physiology due to the ED peptide; however, the ability of the peptide to influence MARCKS phosphorylation or proliferation was not investigated (14). Dose titration determined that $6.25 \mu \mathrm{M}$ was the ideal concentration (data not shown). A549, H1792 and H1975 lung cancer cell lines were cultured without peptide, with $6.25 \mu \mathrm{M}$ control peptide or with $6.25 \mu \mathrm{M}$ ED mimetic peptide. Western blot analysis revealed that the ED peptide mimetic was able to decrease the level of phosphorylated MARCKS in the three lung cancer cell lines (Fig. 2A). Decreasing the level of phosphorylated MARCKS may lead to increased plasma membrane-associated MARCKS and decreased Akt activation. Treatment of A549, and to a lesser extent, H1975, cells with the ED-peptide was able to decrease levels of Akt phosphorylated at the serine 473 and threonine 308 residues (Fig. 2A). In addition, the ED-peptide was able to reduce the total level of Akt, which was most pronounced in the H1792 cells (Fig. 2A). Notably, minimal phosphorylated T308 Akt was observed in the H1792 cells in all conditions, though phosphorylated serine 473 Akt was detectable and was mildly suppressed with the ED-peptide. The effect of MARCKS ED peptide on lung tumor growth was investigated. ATP levels in A549, H1972 and H1975 cell lines treated with the ED peptide mimetic were normalized to ATP levels in lung cancer cells with no peptide intervention. A significant decrease in ATP levels in the A549 $(\mathrm{P}=0.045)$, H1792 $(\mathrm{P}=0.000002)$ and H1975 $(\mathrm{P}=0.000003)$ lung cancer cells following 4 days of treatment with the ED peptide was observed compared with the control cells (Fig. 2B).

MARCKS targeted peptide increases the sensitivity of lung cancer cells to radiation and increases $\gamma H 2 A X$ foci staining. Previously, lentiviral manipulation was used to overexpress NP-MARCKS in A549 cells and their sensitivity to radiation increased (14). In the present study, the effect of treatment with the MARCKS ED peptide mimetic on the radiation sensitivity of lung cancer cells was analyzed. A549, H1792 and H1975 cells were cultured with either the control or ED peptides $1 \mathrm{~h}$ prior to treatment with ionizing radiation. The three cell lines exhibited significantly decreased survival 

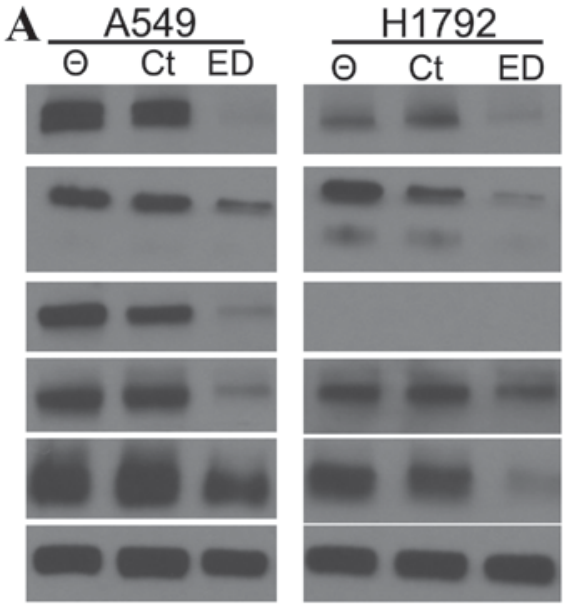

B

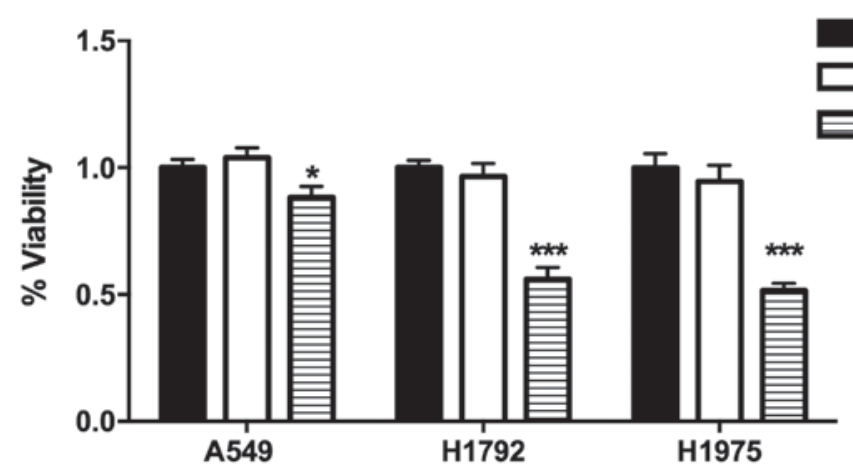

P-MARCKS

MARCKS

T308-Akt

S473-Akt

Akt

Actin

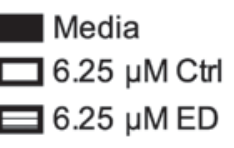
$6.25 \mu \mathrm{M}$ control peptide, or $6.25 \mu \mathrm{M}$ ED peptide. Western blot analysis was used to probe for phosphorylated MARCKS, MARCKS, phosphorylated Akt (T308), phosphorylated Akt (S473), Akt and broad range actin. (B) ATPlite assay measured ATP levels 4 days following A549, H1792 and H1975 cell transfection with no peptide, $6.25 \mu \mathrm{M}$ control peptide, or $6.25 \mu \mathrm{M}$ ED peptide. ${ }^{*} \mathrm{P}<0.05$ compared with Ctrl; ${ }^{* * *} \mathrm{P}<0.0001$ compared with Ctrl. $\Theta$ indicates treatment without peptide. MARCKS, myristoylated alanine rich C-kinase substrate; Ctrl, control; ED, effector domain; Akt, Akt serine/threonine kinase 1; P, phosphorylated.

following ED peptide treatment compared with the control group at irradiation levels 0,5 and 8 Gy (Fig. 3A; $\mathrm{P}<0.05$ ). DNA double-strand breaks in A549 and H1975 cells were examined by measuring $\gamma \mathrm{H} 2 \mathrm{AX}$ foci (22). $\gamma \mathrm{H} 2 \mathrm{AX}$ foci increased in all groups $1 \mathrm{~h}$ following irradiation; however, $24 \mathrm{~h}$ following irradiation A549 and H1975 cells treated with the control peptide exhibited basal level $\gamma \mathrm{H} 2 \mathrm{AX}$ foci staining. Conversely, A549 and H1975 cells treated with the ED peptide demonstrated persistent $\gamma \mathrm{H} 2 \mathrm{AX}$ foci staining (Fig. 3B; $\mathrm{P}<0.01$ compared with the control). The results of the present study suggest that altering the phosphorylation status of MARCKS ED increases lung cell sensitivity to radiation and prolongs DNA damage following irradiation.

\section{Discussion}

MARCKS appears to have tumor suppressive properties in glioblastoma and colon cancer $(5,32)$, however, may function as a tumor promoter in breast and cholangiocarcinoma cancers $(33,34)$. The exact role MARCKS serves in cancer biology remains unclear. The subcellular localization and phosphorylation status of MARCKS ED are important in the normal biological and oncogenic function and regulation of MARCKS $(25,35)$, particularly in lung cancer (36).
Hanada et al (37) reported that squamous cell carcinoma with increased MARCKS expression indicated poor prognosis, however subcellular localization and phosphorylation status were not investigated. Chen et al (38) demonstrated that MARCKS phosphorylation is involved in invasiveness and that lung tumors with elevated and phosphorylated MARCKS exhibited increased invasion in vitro and in patients. Recently, Chen et al (36) demonstrated that increased levels of phosphorylated MARCKS are associated with decreased patient survival and increased tumor growth. A similar 25-amino acid peptide was used to modulate MARCKS phosphorylation and led to reduce migration (36). Previously, the present authors demonstrated that the phosphorylation status of MARCKS is important in subcellular localization, drug sensitivity and the DNA damage response (14). Specifically, lentivirally manipulated A549 lung cancer cell lines were used and showed that NP-MARCKS-expressing cells exhibited increased levels of MARCKS fluorescent staining around the plasma membrane compared with the WT-MARCKS-expressing cells, suggesting ED phosphorylation status may impact MARCKS location (14). In the present study, A549 NP-MARCKS-expressing cells exhibited increased sensitivity to radiation and prolonged double-strand DNA breaks, when assessed using $\gamma \mathrm{H} 2 \mathrm{AX}$ 

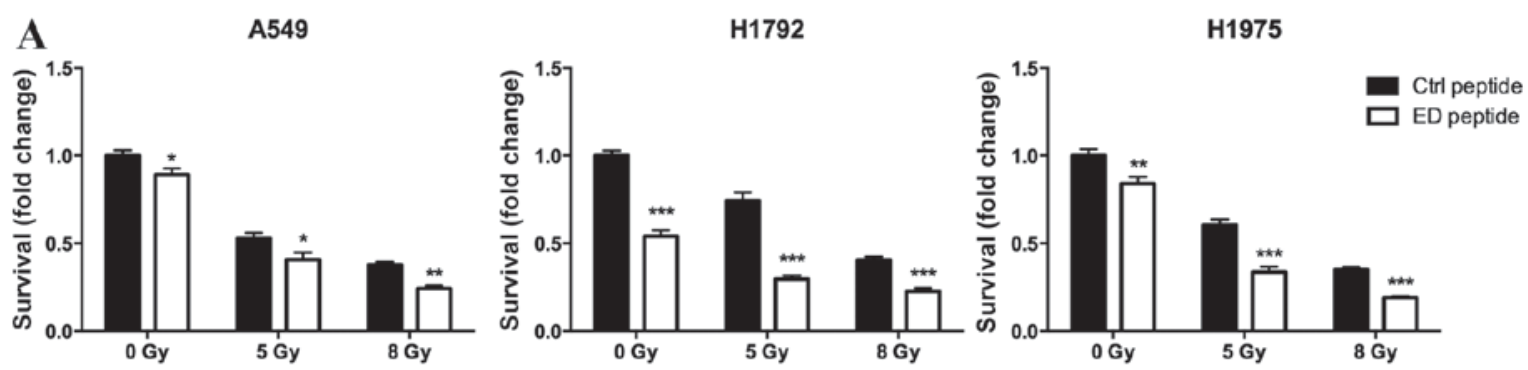

B

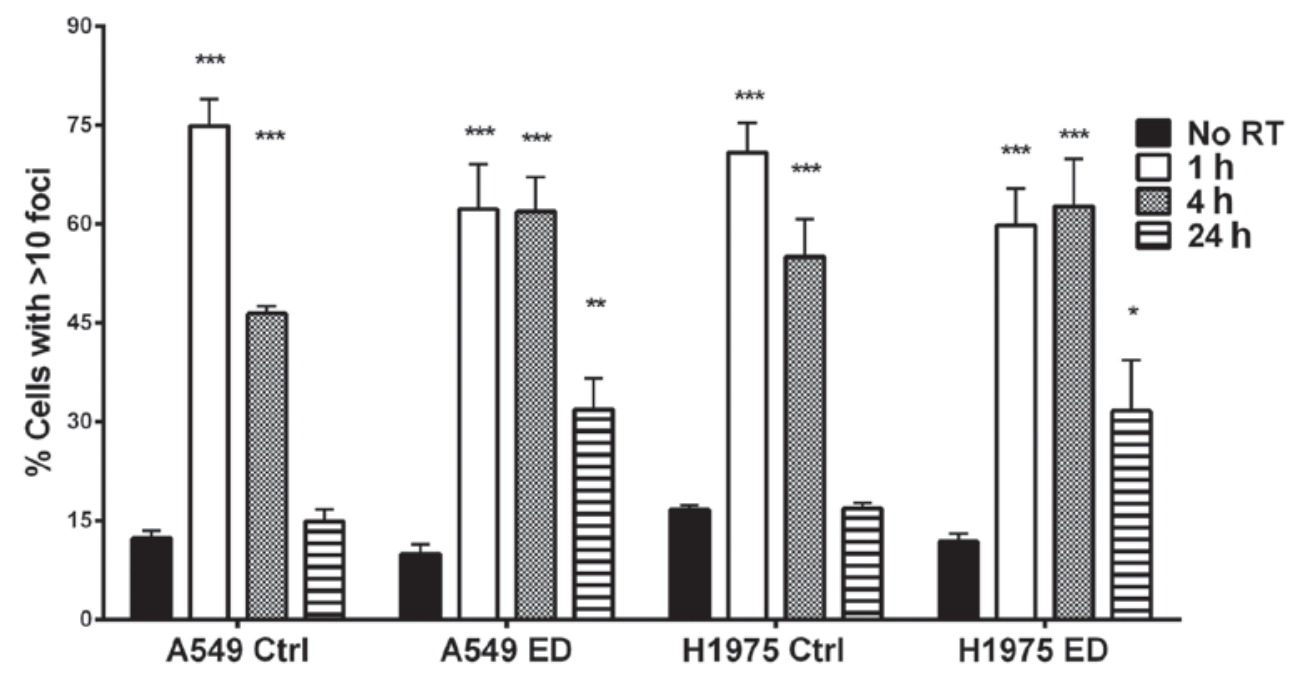

Figure 3. MARCKS targeted peptide therapy alters lung cancer response to ionizing radiation. (A) A549, H1792 and H1975 lung cancer cells were treated with $6.25 \mu \mathrm{M}$ Ctrl peptide or $6.25 \mu \mathrm{M}$ ED peptide $1 \mathrm{~h}$ prior to ionizing irradiation. A total of 4 days following irradiation ATP levels were measured. ATP levels for each cell line were normalized to the cells treated with $0 \mathrm{~Gy}$ and the Ctrl peptide. (B) Cells containing $>10 \gamma \mathrm{H} 2 \mathrm{AX}$ staining foci were counted. A549 and $\mathrm{H} 1975$ cells were cultured with $6.25 \mu \mathrm{M}$ Ctrl peptide or $6.25 \mu \mathrm{M}$ ED peptide prior to $8 \mathrm{~Gy}$ irradiation. Time points post irradiation are indicated. "P $<0.05$ compared with $\mathrm{Ctrl} ;{ }^{* *} \mathrm{P}<0.01$ compared with $\mathrm{Ctrl} ;{ }^{* * *} \mathrm{P}<0.001$ compared with Ctrl. Values shown are means derived from two independent experiments. MARCKS, myristoylated alanine rich C-kinase substrate; Ctrl, control; ED, effector domain; RT, radiation.

foci staining. A549 lung cancer cells are typically inherently resistant to radiation (39). The present results demonstrated that the phosphorylation status of MARCKS is essential to radiation sensitivity. As MARCKS lacks endogenous enzymatic activity, a peptide mimetic was designed to modulate MARCKS phosphorylation. The 25-amino acid sequence of MARCKS ED was conjugated to the HIV TAT peptide, which increased the ability of the peptide to enter the cell.

In the present study, the importance of MARCKS ED phosphorylation status in lung cancer biology was further characterized and the therapeutic benefit of ED peptide treatment in lung cancer was investigated. RNA expression was observed to be altered dependent on the phosphorylation status of MARCKS in lentivirally-engineered A549 cells. Furthermore, the phosphorylation status of MARCKS ED in vivo was observed to impact on lung tumor growth. The efficacy of the ED peptide was tested. Parental A549, H1792 and H1975 lung cancer cells treated with the ED peptide exhibited a decrease in MARCKS phosphorylation. In addition, a decrease in T308 and S473 phosphorylation and total Akt levels was observed. While it is unclear why T308 phosphorylation was so low in the H1792 cells, the
ED peptide was able to decrease A549, H1792 and H1975 proliferation, and increased radiation sensitivity, though it caused prolonged double-strand DNA breaks only in A549 and H1975 cells following irradiation.

Lung cancer is the leading cause of cancer-associated mortality in the United States, and despite targeted therapy and early detection, drug resistance frequently develops (40). Additionally, resistance to ionizing radiation has been observed (41). Targeting MARCKS has the potential to provide a novel mechanism for targeting the RTK-PI3K-Akt signaling cascade. The ED peptide may be able to modulate radiation sensitivity and serve as a future method of avoiding drug resistance (42).

\section{Acknowledgements}

The present study was supported by a Research Scholar Grant from the American Cancer Society (grant no. RSG-14-071-01-TBG), a pilot grant from P20 from the National Cancer Institute of the National Institutes of Health (grant no. CA151129-02), the 2008 ASTRO Junior Faculty Training Research Award and the UAB's Radiation Oncology Pilot Grant. 


\section{References}

1. Siegel R, Naishadham D and Jemal A: Cancer statistics, 2013. CA Cancer J Clin 63: 11-30, 2013.

2. Herbst RS, Heymach JV and Lippman SM: Lung cancer. N Engl J Med 359: 1367-1380, 2008.

3. Engelman JA: Targeting PI3K signalling in cancer: Opportunities, challenges and limitations. Nat Rev Cancer 9: 550-562, 2009.

4. Davies MA: Regulation, role, and targeting of Akt in cancer. J Clin Oncol 29: 4715-4717, 2011.

5. Jarboe JS, Anderson JC, Duarte CW, Mehta T, Nowsheen S, Hicks PH, Whitley AC, Rohrbach TD, McCubrey RO, Chiu S, et al MARCKS regulates growth and radiation sensitivity and is a nove prognostic factor for glioma. Clin Cancer Res 18: 3030-3041, 2012.

6. Glaser M, Wanaski S, Buser CA, Boguslavsky V, Rashidzada W, Morris A, Rebecchi M, Scarlata SF, Runnels LW, Prestwich GD, et al: Myristoylated alanine-rich C kinase substrate (MARCKS) produces reversible inhibition of phospholipase $C$ by sequestering phosphatidylinositol 4,5-bisphosphate in lateral domains. J Biol Chem 271: 26187-26193, 1996.

7. Aderem A: Signal transduction and the actin cytoskeleton: The roles of MARCKS and profilin. Trends Biochem Sci 17: 438-443, 1992.

8. Stumpo DJ, Graff JM, Albert KA, Greengard P and Blackshear PJ: Nucleotide sequence of a cDNA for the bovine myristoylated alanine-rich C kinase substrate (MARCKS). Nucleic Acids Res 17: 3987-3988, 1989.

9. Heemskerk FM, Chen HC and Huang FL: Protein kinase C phosphorylates Ser152, Ser156 and Ser163 but not Ser160 of MARCKS in rat brain. Biochem Biophys Res Commun 190: 236-241, 1993.

10. Gambhir A, Hangyás-Mihályné G, Zaitseva I, Cafiso DS, Wang J, Murray D, Pentyala SN, Smith SO and McLaughlin S: Electrostatic sequestration of PIP2 on phospholipid membranes by basic/aromatic regions of proteins. Biophys J 86: 2188-2207, 2004

11. Wang J, Arbuzova A, Hangyás-Mihályné G and McLaughlin S: The effector domain of myristoylated alanine-rich $C$ kinase substrate binds strongly to phosphatidylinositol 4,5-bisphosphate. J Biol Chem 276: 5012-5019, 2001.

12. McLaughlin S and Aderem A: The myristoyl-electrostatic switch: A modulator of reversible protein-membrane interactions. Trends Biochem Sci 20: 272-276, 1995.

13. Manenti S, Sorokine O, Van Dorsselaer A and Taniguchi H: Affinity purification and characterization of myristoylated alanine-rich protein kinase $\mathrm{C}$ substrate (MARCKS) from bovine brain. Comparison of the cytoplasmic and the membrane-bound forms. J Biol Chem 267: 22310-22315, 1992.

14. Rohrbach TD, Jarboe JS, Anderson JC, Trummell HQ, Hicks PH, Weaver AN, Yang ES, Oster RA, Deshane JS, Steele C, et al: Targeting the effector domain of the myristoylated alanine rich $\mathrm{C}$-kinase substrate enhances lung cancer radiation sensitivity. Int J Oncol 46: 1079-1088, 2015

15. Graff JM, Rajan RR, Randall RR, Nairn AC and Blackshear PJ: Protein kinase $\mathrm{C}$ substrate and inhibitor characteristics of peptides derived from the myristoylated alanine-rich $\mathrm{C}$ kinase substrate (MARCKS) protein phosphorylation site domain. J Biol Chem 266: 14390-14398, 1991.

16. Isayeva T, Xu J, Ragin C, Dai Q, Cooper T, Carroll W, Dayan D, Vered M, Wenig B, Rosenthal E, et al: The protective effect of p16(INK4a) in oral cavity carcinomas: p16 (Ink4A) dampens tumor invasion-integrated analysis of expression and kinomics pathways. Mod Pathol 28: 631-653, 2015.

17. Malkov VA, Serikawa KA, Balantac N, Watters J, Geiss G, Mashadi-Hossein A and Fare T: Multiplexed measurements of gene signatures in different analytes using the Nanostring nCounter Assay System. BMC Res Notes 2: 80, 2009.

18. Willey CD, Xiao D, Tu T, Kim KW, Moretti L, Niermann KJ, Tawtawy MN, Quarles CC and Lu B: Enzastaurin (LY317615), a protein kinase $\mathrm{C}$ beta selective inhibitor, enhances antiangiogenic effect of radiation. Int J Radiat Oncol Biol Phys 77: 1518-1526, 2010.

19. Cawthorne C, Swindell R, Stratford IJ, Dive C and Welman A: Comparison of doxycycline delivery methods for Tet-inducible gene expression in a subcutaneous xenograft model. J Biomol Tech 18: 120-123, 2007

20. Ayers GD, McKinley ET, Zhao P, Fritz JM, Metry RE, Deal BC, Adlerz KM, Coffey RJ and Manning HC: Volume of preclinical xenograft tumors is more accurately assessed by ultrasound imaging than manual caliper measurements. J Ultrasound Med 29: 891-901, 2010
21. Jensen MM, Jørgensen JT, Binderup T and Kjaer A: Tumor volume in subcutaneous mouse xenografts measured by microCT is more accurate and reproducible than determined by 18F-FDG-microPET or external caliper. BMC Med Imaging 8: 16,2008

22. Yang ES, Wang H, Jiang G, Nowsheen S, Fu A, Hallahan DE and Xia F: Lithium-mediated protection of hippocampal cells involves enhancement of DNA-PK-dependent repair in mice. J Clin Invest 119: 1124-1135, 2009.

23. Nowsheen S, Bonner JA and Yang ES: The poly (ADP-Ribose) polymerase inhibitor ABT-888 reduces radiation-induced nuclear EGFR and augments head and neck tumor response to radiotherapy. Radiother Oncol 99: 331-338, 2011.

24. Yang ES, Nowsheen S, Wang T, Thotala DK and Xia F: Glycogen synthase kinase 3beta inhibition enhances repair of DNA double-strand breaks in irradiated hippocampal neurons. Neuro Oncol 13: 459-470, 2011.

25. Arbuzova A, Schmitz AA and Vergères G: Cross-talk unfolded: MARCKS proteins. Biochem J 362: 1-12, 2002

26. Seykora JT, Myat MM, Allen LA, Ravetch JV and Aderem A: Molecular determinants of the myristoyl-electrostatic switch of MARCKS. J Biol Chem 271: 18797-18802, 1996.

27. Gialeli C, Theocharis AD and Karamanos NK: Roles of matrix metalloproteinases in cancer progression and their pharmacological targeting. FEBS J 278: 16-27, 2011

28. Witsch E, Sela M and Yarden Y: Roles for growth factors in cancer progression. Physiology (Bethesda) 25: 85-101, 2010.

29. Cascorbi I, Brockmöller J and Roots I: A C4887A polymorphism in exon 7 of human CYP1A1: Population frequency, mutation linkages, and impact on lung cancer susceptibility. Cancer Res 56: 4965-4969, 1996.

30. Ma L, Young J, Prabhala H, Pan E, Mestdagh P, Muth D, Teruya-Feldstein J, Reinhardt F, Onder TT, Valastyan S, et al: miR-9, a MYC/MYCN-activated microRNA, regulates E-cadherin and cancer metastasis. Nat Cell Biol 12: 247-256, 2010

31. Westhoff B, Colaluca IN, D'Ario G, Donzelli M, Tosoni D, Volorio S, Pelosi G, Spaggiari L, Mazzarol G, Viale G, et al: Alterations of the Notch pathway in lung cancer. Proc Natl Acad Sci USA 106: 22293-22298, 2009.

32. Bickeboller M, Tagscherer KE, Kloor M, Jansen L, Chang-Claude J, Brenner H, Hoffmeister M, Toth C, Schirmacher P, Roth W and Bläker H: Functional characterization of the tumor-suppressor MARCKS in colorectal cancer and its association with survival. Oncogene 34: 1150-1159, 2015.

33. Browne BC, Hochgräfe F, Wu J, Millar EK, Barraclough J, Stone A, McCloy RA, Lee CS, Roberts C, Ali NA, et al: Global characterization of signalling networks associated with tamoxifen resistance in breast cancer. FEBS J 280: 5237-5257, 2013.

34. Techasen A,Loilome W, NamwatN, et al: Myristoylated alanine-rich $\mathrm{C}$ kinase substrate phosphorylation promotes cholangiocarcinoma cell migration and metastasis via the protein kinase $\mathrm{C}$-dependent pathway. In: Cancer Sci, England, pp658-pp665, 2010.

35. Wright PE and Dyson HJ: Intrinsically unstructured proteins: Re-assessing the protein structure-function paradigm. J Mol Biol 293: 321-331, 1999

36. Chen $\mathrm{CH}$, Chiu $\mathrm{CL}$, Adler KB and Wu R: A novel predictor of cancer malignancy: Up-regulation of myristoylated alanine-rich $\mathrm{C}$ kinase substrate phosphorylation in lung cancer. Am J Respir Crit Care Med 189: 1002-1004, 2014

37. Hanada S, Kakehashi A, Nishiyama N, Wei M, Yamano S, Chung K, Komatsu H, Inoue H, Suehiro S and Wanibuchi H: Myristoylated alanine-rich C-kinase substrate as a prognostic biomarker in human primary lung squamous cell carcinoma. Cancer Biomark 13: 289-298, 2013.

38. Chen CH, Thai P, Yoneda K, Adler KB, Yang PC and Wu R: A peptide that inhibits function of Myristoylated Alanine-Rich C Kinase Substrate (MARCKS) reduces lung cancer metastasis. Oncogene 33: 3696-3706, 2014.

39. Yang HJ, Kim N, Seong KM, Youn H and Youn B: Investigation of radiation-induced transcriptome profile of radioresistant non-small cell lung cancer A549 cells using RNA-seq. PloS One 8: e59319, 2013.

40. Shanker M, Willcutts D and Roth JA: Drug resistance in lung cancer. Lung Cancer: Targets and Therapy 1: 23-36, 2010.

41. Schuurbiers OC, Kaanders JH, van der Heijden HF, Dekhuijzen RP, Oyen WJ and Bussink J: The PI3-K/AKT-pathway and radiation resistance mechanisms in non-small cell lung cancer. $\mathrm{J}$ Thorac Oncol 4: 761-767, 2009.

42. Garraway LA and Jänne PA: Circumventing cancer drug resistance in the era of personalized medicine. Cancer Discov 2: 214-226, 2012. 